Commun. Fac. Sci. Univ. Ank. Ser. A1 Math. Stat.

Volume 68, Number 2, Pages 1761-1773(2019)

DOI: $10.31801 /$ cfsuasmas.444554

ISSN 1303-5991 E-ISSN 2618-6470

http://communications.science.ankara.edu.tr/index.php?series=A1

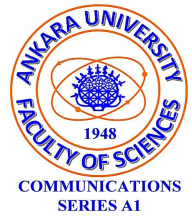

\title{
HENNEBERG'S ALGEBRAIC SURFACES IN MINKOWSKI
} 3-SPACE

\author{
ERHAN GÜLER AND VAHIT ZAMBAK
}

\begin{abstract}
We consider the Henneberg's algebraic zero mean curvature surfaces in three dimensional Minkowski space, and compute their classes, degrees and integral free representations. We also draw some figures of the algebraic surfaces.
\end{abstract}

\section{INTRODUCTION}

Classical and first non-orientable minimal surface in $\mathbb{E}^{3}$ were introduced by Henneberg [4, [5].

After Weierstrass' representation in [13] in three dimensional Euclidean space $\mathbb{E}^{3}$, Kobayashi 8 gave an analogous Weierstrass-type representation for conformal spacelike surfaces with mean curvature identically 0 , called maximal surfaces, in 3 -dimensional Minkowski space $\mathbb{E}^{2,1}$. Unlike the case of minimal surfaces in $\mathbb{E}^{3}$, maximal surfaces have singularities, generally. Details about singularities of maximal surfaces can be found in 2, 12. We remark that Magid [9] gave a Weierstrasstype representation for timelike surfaces with mean curvature identically 0 , called timelike minimal surfaces, in $\mathbb{E}^{2,1}$, see also [7].

In Section 2, we give the Henneberg minimal surface in $(u, v)$ coordinates via Weierstrass representation in $\mathbb{E}^{3}$. In Section 3 , we compute algebraic equation defining Henneberg surface $\mathcal{H}(u, v)$ in terms of running coordinates, and obtain class and degree of it. In Section 4 using integral free form of Weierstrass, we give an algebraic function of Henneberg surface. We review Weierstrass-type representations for spacelike maximal and timelike minimal surfaces in $\mathbb{E}^{2,1}$ in Section 5 of this paper, and give explicit parametrizations for spacelike maximal and timelike minimal Henneberg surfaces. We calculate algebraic surfaces of the spacelike maximal and timelike minimal Henneberg surfaces in terms of their coordinates in Section 6. Then give degrees and classes of the surfaces. Finally, we find some algebraic

Received by the editors: July 17, 2018; Accepted: March 06, 2019.

2010 Mathematics Subject Classification. Primary 53A35; Secondary 65D18.

Key words and phrases. Weierstrass representation, spacelike maximal Henneberg surface, timelike minimal Henneberg surface, degree, class, integral free representation. 
functions for spacelike maximal and timelike minimal Henneberg surfaces by using integral free forms in Section 7.

\section{Henneberg ZMC Surface}

Let $\mathbb{E}^{3}$ be three dimensional Euclidean space with natural metric $\langle.,\rangle=.d x^{2}+$ $d y^{2}+d z^{2}$. We will often identify $\vec{x}$ and $\overrightarrow{x^{t}}$ without further comment.

Let $\mathcal{U}$ be an open subset of $\mathbb{C}$. A minimal (or isotropic) curve is an analytic function $\vartheta: \mathcal{U} \rightarrow \mathbb{C}^{n}$ such that $\vartheta^{\prime}(\zeta) \cdot \vartheta^{\prime}(\zeta)=0$, where $\zeta \in \mathcal{U}$, and $\vartheta^{\prime}:=\frac{\partial \vartheta}{\partial \zeta}$.

In addition, if $\vartheta^{\prime} \cdot \overline{\vartheta^{\prime}}=\left|\vartheta^{\prime}\right|^{2} \neq 0$, then $\vartheta$ is a regular minimal curve. We then have minimal surfaces in the associated family of a minimal curve, like as given by the following Weierstrass representation theorem for ZMC (zero mean curvature) surfaces, or minimal surfaces, shortly.

Theorem 1. (K. Weierstrass [13]). Let $g(\omega)$ be a meromorphic function and let $f(\omega)$ be a holomorphic function, $f g^{2}$ is analytic, defined on a simply connected open subset $\mathcal{U} \subset \mathbb{C}$ such that $f(\omega)$ does not vanish on $\mathcal{U}$ except at the poles of $g(\omega)$. Then

$$
\mathbf{x}(u, v)=\operatorname{Re} \int^{\zeta}\left(\begin{array}{c}
f\left(1-g^{2}\right) \\
i f\left(1+g^{2}\right) \\
2 f g
\end{array}\right) d \omega, \quad(\zeta=u+i v)
$$

is a conformal immersion with mean curvature identically 0 (i.e. conformal minimal surface). Conversely, any conformal minimal surface can be described in this manner.

Remark 2. A pair of a meromorphic function $g$ and a holomorphic function $f$, $(f, g)$ is called Weierstrass data for a minimal surface.

Lemma 3. The curve of Henneberg

$$
\hbar(\zeta)=\left(-\frac{1}{3}\left(\zeta^{3}-\frac{1}{\zeta^{3}}\right)+\zeta-\frac{1}{\zeta}, i\left(\frac{1}{3}\left(\zeta^{3}+\frac{1}{\zeta^{3}}\right)+\zeta+\frac{1}{\zeta}\right), \zeta^{2}+\frac{1}{\zeta^{2}}\right)
$$

is a minimal curve in $\mathbb{C}^{3}, \zeta \in \mathbb{C}-\{0\}, i=\sqrt{-1}$.

So, we have $\hbar^{\prime} \cdot \hbar^{\prime}=0$. Hence, in $\mathbb{E}^{3}$, Henneberg surface is as follows

$$
\mathcal{H}(u, v)=\operatorname{Re} \int \hbar^{\prime}(\zeta) d \zeta
$$

where $\zeta=u+i v$. Therefore, $\operatorname{Im} \int \hbar^{\prime}(\zeta) d \zeta$ gives adjoint surface $\mathcal{H}^{*}(u, v)$ of Henneberg surface $\mathcal{H}(u, v)$ in (3).

Remark 4. For the first element of (1), we write the following

$$
\operatorname{Re} \int^{\zeta} f(\omega)\left(1-\omega^{2}\right) d \omega(+c)=\operatorname{Re} \int^{-1 / \bar{\zeta}} \widetilde{f}(\omega)\left(1-\omega^{2}\right) d \omega
$$




$$
\begin{aligned}
& =\operatorname{Re}\left(\overline{\int^{-1 / \bar{\zeta}} \tilde{f}(\omega)\left(1-\omega^{2}\right) d \omega}\right) \\
& =\operatorname{Re} \int^{-1 / \zeta} \overline{\widetilde{f}(\bar{\omega})\left(1-\bar{\omega}^{2}\right)} d \omega \\
& =\operatorname{Re} \int^{-1 / \zeta} \overline{\widetilde{f}(\bar{\omega})}\left(1-\omega^{2}\right) d \omega \\
& =\operatorname{Re} \int^{\zeta} \overline{\tilde{f}\left(-\frac{1}{\bar{\omega}}\right)}\left(1-\frac{1}{\omega^{2}} \frac{1}{\omega^{2}}\right) d \omega,
\end{aligned}
$$

and can also write for other two equations, where

$$
\tilde{f}(\zeta)=-\frac{1}{\zeta^{4}} \overline{f\left(-\frac{1}{\bar{\zeta}}\right)}
$$

Here, $\tilde{f}$ gives the same surface as $f$, but it induces the opposite orientation on surface.

See 11 for details. So, we see the Weierstrass data of Henneberg surface:

Lemma 5. The Weierstrass data determined by the functions

$$
(f, g)=\left(1-\zeta^{-4}, \zeta\right)
$$

is a representation of Henneberg surface on $\mathbb{C}-\{0\}$.

Now, we give a Lemma about a complete surface:

Lemma 6. ([1]) An immersed surface $M$ is complete iff any closed bounded subset of $M$ is compact.

When we allow the existence of singular points on minimal surfaces, then that kind surfaces will be called generalized minimal surfaces. Singular points of generalized minimal surfaces have a special form, and then they have acquired a special name called branch points. The condition that $\zeta_{0}$ is a branch point is equivalent to $f\left(\zeta_{0}\right)=0$ and $f g^{2}\left(\zeta_{0}\right)=0$. Henneberg surface is a non-orientable minimal surface since it has a Möbius strip. The branched immersion of an infinite Möbius strip as a complete (see Lemma 3) minimal surface has been known for a long time - it is the Henneberg surface. The branch points are the fourth roots of unity, that is, $\zeta= \pm i, \pm 1$. By using the stereographic projection, we can identify $\mathbb{C}-\{0, \pm i, \pm 1\}$ with $S^{2}$ minus three pairs of antipodal points, the points $\pm i, \pm 1$ occurring on the equator of $S^{2}$. Therefore, Henneberg surface is the image of the projective plane punctured at three points. See [1] and also [11] for details.

So, throughout the paper, we will consider Henneberg surface has four branch points, and defined on $\mathbb{C}-\{0\}$. 
In 1875, in his doctorate thesis 4, Henneberg gave his classical minimal surface using polar coordinates, as follows:

$$
\mathcal{H}(r, q)=\left(\begin{array}{c}
\frac{r}{2}\left[\left(r^{2}+3\right) \sin (3 q)+3 \sin (q)\right] \\
-\frac{r}{2}\left[\left(r^{2}+3\right) \cos (3 q)-3 \cos (q)\right] \\
\frac{3}{2}\left(r^{2}+2\right) \cos (2 q)
\end{array}\right)
$$

See also [3, 5, 6, 10, for details.

Throughout this work, apart from polar form of Henneberg, we study Henneberg surface in $(u, v)$ coordinates, taking $\zeta=u+i v$ at Cartesian coordinates $x, y, z$, and also at inhomogeneous tangential coordinates $a, b, c$ by using Weierstrass representation equation.

Next, we give a theorem about minimality of surface $\mathcal{H}(u, v)$.

Theorem 7. Henneberg's surface

$$
\mathcal{H}(u, v)=\left(\begin{array}{c}
u+u v^{2}-\frac{u^{3}}{3}-\frac{u}{u^{2}+v^{2}}-\frac{u v^{2}}{\left(u^{2}+v^{2}\right)^{3}}+\frac{u^{3}}{3\left(u^{2}+v^{2}\right)^{3}} \\
u-u^{2} v+\frac{v^{3}}{3}+\frac{u}{u^{2}+v^{2}}+\frac{u^{2} v}{\left(u^{2}+v^{2}\right)^{3}}-\frac{v^{3}}{3\left(u^{2}+v^{2}\right)^{3}} \\
u^{2}-v^{2}+\frac{u^{2}-v^{2}}{\left(u^{2}+v^{2}\right)^{2}}
\end{array}\right)=\left(\begin{array}{l}
x(u, v) \\
y(u, v) \\
z(u, v)
\end{array}\right)
$$

is a minimal surface in $\mathbb{E}^{3}$.

Proof. The coefficients of the first fundamental form of the Henneberg's surface $\mathcal{H}(u, v)(\mathcal{H}$, for short) are

$$
\begin{aligned}
& E=\lambda^{-4}(\lambda+1)^{2}\left((\lambda+1)^{2}-4 u^{2}\right)\left(u^{2}+(v+1)^{2}\right)\left(u^{2}+(v-1)^{2}\right)=G, \\
& F=0,
\end{aligned}
$$

and its Gauss map is as follows

$$
e=\left(\frac{2 u}{\lambda+1}, \frac{2 v}{\lambda+1}, \frac{\lambda-1}{\lambda+1}\right)
$$

where $\lambda=u^{2}+v^{2}$. The coefficients of the second fundamental form of $\mathcal{H}$ are as follows

$$
L=-2 \lambda^{-4}\left(\lambda^{4}-\left(\lambda^{2}-8 u^{2} v^{2}\right)\right)=-N, \quad M=8 \lambda^{-4}\left(u^{2}-v^{2}\right) u v .
$$

We have

$$
\operatorname{det} I I=-4 \lambda^{-4}\left((\lambda+1)^{2}-4 u^{2}\right)\left(u^{2}+(v+1)^{2}\right)\left(u^{2}+(v-1)^{2}\right) .
$$

Hence, we get the mean curvature and the Gaussian curvature of $\mathcal{H}$, as follows

$$
\begin{aligned}
& H=0, \\
& K=-\frac{4 \lambda^{4}}{(\lambda+1)^{4}\left((\lambda+1)^{2}-4 u^{2}\right)\left(u^{2}+(v+1)^{2}\right)\left(u^{2}+(v-1)^{2}\right)},
\end{aligned}
$$

respectively. This completes the proof. 
Next, by using Sylvester elimination method, we calculate the implicit equation, degree and class of Henneberg minimal surface $\mathcal{H}(u, v)$.

\section{Degree and Class of Henneberg Surface in $\mathbb{E}^{3}$}

The set of roots of a polynomial $Q(x, y, z)=0$ gives an algebraic surface. An algebraic surface $\mathbf{s}$ is said to be of degree $\mathbf{d}$ when $\mathbf{d}=\operatorname{deg}(\mathbf{s})$.

The tangent plane at a point $(u, v)$ on a surface $\mathbf{s}(u, v)=(x(u, v), y(u, v)$, $z(u, v))$ is given by

$$
X x+Y y+Z z+P=0,
$$

where the Gauss map is $e=(X(u, v), Y(u, v), Z(u, v))$, and $P=P(u, v)$. Then, we have inhomogeneous tangential coordinates

$$
(a, b, c)=(X / P, Y / P, Z / P) .
$$

At a point $(u, v)$ on a surface, we only have one tangent plane. So, we can obtain an implicit equation $\hat{Q}(a, b, c)=0$ of $\mathbf{s}(u, v)$ in inhomogeneous tangential coordinates, and the maximum degree of the equation gives the class of $\mathbf{s}(u, v)$. See [10, for details.

It is known that the surface $\mathcal{H}(r, q)$ in (4) has class 5 and degree 15 . So, it is also an algebraic minimal surface. See [4, 5, 10, for expanded results.

Using Sylvester elimination technique, we find the irreducible implicit equation of surface $\mathcal{H}(u, v)$ in (5) as follows:

$Q(x, y, z)=64 x^{6} z^{9}+192 x^{4} y^{2} z^{9}+192 x^{2} y^{4} z^{9}+64 y^{6} z^{9}-432 x^{8} z^{6}-864 x^{6} y^{2} z^{6}-$ $768 x^{4} z^{10}+864 x^{2} y^{6} z^{6}+432 y z^{6}+768 y z^{10}-1215 x^{10} z^{3}-9963 x^{8} y z^{3}-3888 x^{8} z^{5}-$ $23814 x^{6} y^{4} z^{3}-15552 x^{6} y^{2} z^{5}+4320 x^{6} z^{7}-23814 x^{4} y^{6} z^{3}-23328 x^{4} y^{4} z^{5}-7776 x^{4} y^{2} z^{7}-$ $768 x^{4} z^{9}-9963 x^{2} y^{8} z^{3}-15552 x^{2} y^{6} z^{5}-7776 x^{2} y^{4} z^{7}+4608 x^{2} y^{2} z^{9}+2304 x^{2} z^{11}-$ $1215 y^{10} z^{3}-3888 y^{8} z^{5}+4320 y^{6} z^{7}-768 y^{4} z^{9}+2304 y^{2} z^{11}-729 x^{12}-4374 x^{10} z^{2}+$ $2187 x^{8} y^{4}-13122 x^{8} y^{2} z^{2}+7776 x^{8} z^{4}-8748 x 6 y^{4} z^{2}+62208 x^{6} y^{2} z^{4}+52032 x^{6} z^{6}-$ $2187 x^{4} y^{8}+8748 x^{4} y^{6} z^{2}+10560 x 4 y^{2} z^{6}-5376 x^{4} z^{8}+13122 x^{2} y^{8} z^{2}-62208 x^{2} y^{6} z^{4}-$ $10560 x^{2} y^{4} z^{6}+4608 x^{2} z^{10}+729 y^{12}+4374 y^{10} z^{2}-7776 y^{8} z^{4}-52032 y^{6} z^{6}+5376 y^{4} z^{8}$ $-4608 y^{2} z^{10}+8748 x^{10} z-26244 x^{8} y^{2} z+69120 x^{8} z^{3}+17496 x^{6} y^{4} z+89856 x^{6} z^{5}+$ $17496 x^{4} y^{6} z-511488 x 4 y^{4} z^{3}-573696 x^{4} y^{2} z^{5}-162816 x^{4} z^{7}-26244 x^{2} y^{8} z-573696 x^{2} y^{4} z^{5}-$ $276480 x^{2} y^{2} z^{7}-27648 x^{2} z^{9}+8748 y^{10} z+69120 y^{8} z^{3}+89856 y^{6} z^{5}-162816 y^{4} z^{7}-$ $27648 y^{2} z^{9}+9720 x^{10}-79704 x^{8} y^{2}+31104 x^{8} z^{2}+190512 x^{6} y^{4}-248832 x^{6} y^{2} z^{2}-$ $179712 x^{6} z^{4}-190512 x^{4} y^{6}-1147392 x^{4} y^{2} z^{4}-595968 x^{4} z^{6}+79704 x^{2} y^{8}+248832 x^{2} y^{6} z^{2}+$ $1147392 x^{2} y^{4} z^{4}-55296 x^{2} z^{8}-9720 y^{10}-31104 y^{8} z^{2}+179712 y^{6} z^{4}+595968 y^{4} z^{6}+$ $55296 y^{2} z^{8}-62208 x^{8} z+248832 x^{6} y^{2} z-416256 x^{6} z^{3}-373248 x^{4} y^{4} z+84480 x^{4} y^{2} z^{3}-$ $651264 x^{4} z^{5}+248832 x^{2} y^{6} z+84480 x^{2} y^{4} z^{3}+1105920 x^{2} y^{2} z^{5}+110592 x^{2} z^{7}-62208 y^{8} z-$ $416256 y^{6} z^{3}-651264 y^{4} z^{5}+110592 y^{2} z^{7}-27648 x^{8}+55296 x^{6} y^{2}-138240 x^{6} z^{2}-$ $248832 x^{4} y^{2} z^{2}-86016 x^{4} z^{4}-55296 x^{2} y^{6}+248832 x^{2} y^{4} z^{2}+221184 x^{2} z^{6}+27648 y^{8}+$ $138240 y^{6} z^{2}+86016 y^{4} z^{4}-221184 y^{2} z^{6}-49152 x^{4} z^{3}-294912 x^{2} y^{2} z^{3}-147456 x^{2} z^{5}-$ $49152 y^{4} z^{3}-147456 y^{2} z^{5}-32768 x^{6}+98304 x^{4} y^{2}-196608 x^{4} z^{2}-98304 x^{2} y^{4}-294912 x^{2} z^{4}+$ $32768 y^{6}+196608 y^{4} z^{2}+294912 y^{2} z^{4}$. 
Its degree is $\operatorname{deg}(\mathcal{H}(u, v))=15$. Therefore, $Q(x, y, z)=0$ is an algebraic minimal surface (see Figure 1, Left) of $\mathcal{H}$.

To find the class of surface $\mathcal{H}$, we use (5), (6) and (7). Hence, we find

$$
P(u, v)=\frac{\varrho(u, v)}{3(\lambda+1) \lambda^{2}},
$$

and then, obtain inhomogeneous tangential coordinates

$$
a=\frac{6 u \lambda^{2}}{\varrho(u, v)}, b=\frac{6 v \lambda^{2}}{\varrho(u, v)}, c=\frac{3(\lambda-1) \lambda^{2}}{\varrho(u, v)},
$$

where $\lambda=u^{2}+v^{2}, \varrho(u, v)=-(\lambda-1)\left(u^{2}-v^{2}\right)\left(\lambda^{2}+4 \lambda+1\right) \neq 0$. In the coordinates $a, b, c$, the irreducible implicit equation $\hat{Q}(a, b, c)=0$ of $\mathcal{H}$ (see Figure 2, Left) is as follows

$$
\hat{Q}(a, b, c)=-6 a^{4} c-4 a^{2} c^{3}+6 b^{4} c+4 b^{2} c^{3}-3 a^{4}-6 a^{2} b^{2}-3 b^{4} .
$$

Therefore, $\hat{Q}(a, b, c)$ is an algebraic Henneberg minimal surface of $\mathcal{H}$, and the class of the surface is $\operatorname{cl}(\mathcal{H}(u, v))=5$.

\section{Integral Free Form in $\mathbb{E}^{3}$}

Integral free form of the Weierstrass representation (1) for minimal surfaces in $\mathbb{E}^{3}$ is

$$
\left(\begin{array}{l}
x \\
y \\
z
\end{array}\right)=\operatorname{Re}\left(\begin{array}{c}
\left(1-w^{2}\right) \phi^{\prime \prime}(w)+2 w \phi^{\prime}(w)-2 \phi(w) \\
i\left[\left(1+w^{2}\right) \phi^{\prime \prime}(w)-2 w \phi^{\prime}(w)+2 \phi(w)\right] \\
2\left[w \phi^{\prime \prime}(w)-\phi^{\prime}(w)\right]
\end{array}\right) \equiv \operatorname{Re}\left(\begin{array}{c}
f_{1}(w) \\
f_{2}(w) \\
f_{3}(w)
\end{array}\right)
$$

where algebraic function $\phi(w)$ and the functions $f_{i}(w)$ are connected by the relation

$$
\phi(w)=\frac{1}{4}\left(w^{2}-1\right) f_{1}(w)-\frac{i}{4}\left(w^{2}+1\right) f_{2}(w)-\frac{1}{2} w f_{3}(w)
$$

for $w \in \mathbb{C}[14$. Integral free form is suitable for algebraic minimal surfaces (see [10]).

Corollary 8. The algebraic function

$$
\phi(w)=\frac{w^{4}+1}{6 w},
$$

leads to Henneberg's minimal surface, where $w \in \mathbb{C}-\{0\}$.

\section{Henneberg Surfaces in $\mathbb{E}^{2,1}$}

Let

$$
\mathbb{E}^{n, 1}:=\left(\left\{x=\left(x_{1}, \cdots, x_{n}, x_{0}\right)^{t} \mid x_{i} \in \mathbb{R}\right\},\langle\cdot, \cdot\rangle\right)
$$

be the $(n+1)$-dimensional Lorentz-Minkowski (for short, Minkowski) space with Lorentz metric $\langle x, y\rangle=x_{1} y_{1}+\cdots+x_{n} y_{n}-x_{0} y_{0}$. A vector $x \in \mathbb{E}^{n, 1}$ is called spacelike if $\langle x, x\rangle>0$, timelike if $\langle x, x\rangle<0$, and lightlike if $x \neq 0$ and $\langle x, x\rangle=0$. 
A surface in $\mathbb{E}^{n, 1}$ is called spacelike (resp. timelike, lightlike) if the induced metric on the tangent planes is a Riemannian (resp. Lorentzian, degenerate) metric.

5.1. Henneberg spacelike maximal surface. Kobayashi 8 found a Weierstrass type representation for spacelike conformal maximal surfaces in $\mathbb{E}^{2,1}$.

Theorem 9. Let $g(\omega)$ be a meromorphic function and let $f(\omega)$ be a holomorphic function, $f g^{2}$ is analytic, defined on a simply connected open subset $\mathcal{U} \subset \mathbb{C}$ such that $f(\omega)$ does not vanish on $\mathcal{U}$ except at the poles of $g(\omega)$. Then

$$
\mathbf{x}(u, v)=\operatorname{Re} \int^{\zeta}\left(\begin{array}{c}
f\left(1+g^{2}\right) \\
i f\left(1-g^{2}\right) \\
2 f g
\end{array}\right) d \omega, \quad(\zeta=u+i v)
$$

is a spacelike conformal immersion with mean curvature identically 0 (i.e. spacelike conformal maximal surface). Conversely, any spacelike conformal maximal surface can be described in this manner.

We call Henneberg's maximal surface given by $(f, g)=\left(1-\zeta^{-4}, \zeta\right)$ the spacelike maximal surface $\mathcal{H}(u, v)$ (spacelike $\mathcal{H}$, for short), where $\widetilde{f}(\zeta)=-\frac{1}{\zeta^{4}} \overline{f\left(-\frac{1}{\bar{\zeta}}\right)}$ as in the Weierstrass representation of the Euclidean case. The parametrization of spacelike $\mathcal{H}$ is

$$
\mathcal{H}(u, v)=\left(\begin{array}{c}
u-u v^{2}+\frac{u^{3}}{3}+\frac{u}{u^{2}+v^{2}}-\frac{u v^{2}}{\left(u^{2}+v^{2}\right)^{3}}+\frac{u^{3}}{3\left(u^{2}+v^{2}\right)^{3}} \\
-v+u^{2} v-\frac{v^{3}}{3}-\frac{v}{u^{2}+v^{2}}+\frac{u^{2} v}{\left(u^{2}+v^{2}\right)^{3}}-\frac{v^{3}}{3\left(u^{2}+v^{2}\right)^{3}} \\
u^{2}-v^{2}+\frac{u^{2}-v^{2}}{\left(u^{2}+v^{2}\right)^{2}}
\end{array}\right)=\left(\begin{array}{l}
x(u, v) \\
y(u, v) \\
z(u, v)
\end{array}\right)
$$

with Gauss map

$$
e=\left(\frac{2 u}{\lambda-1}, \frac{2 v}{\lambda-1}, \frac{\lambda+1}{\lambda-1}\right)
$$

where $\lambda=u^{2}+v^{2} \neq 1$.

5.2. Henneberg timelike minimal surface. Next, we give the Weierstrass type representation for timelike minimal surfaces in $\mathbb{E}^{2,1}$, which was obtained by $M$. Magid [9] (see also [7]).

Theorem 10. Let $f_{1}(u), g_{1}(u)$ (resp. $f_{2}(v), g_{2}(v)$ ) be smooth functions depending on only u (resp. v) on a connected orientable 2-manifold with local coordinates $u$, $v$. Then

$$
\widehat{\mathbf{x}}(u, v)=\int\left(\begin{array}{c}
2 f_{1} g_{1} \\
f_{1}\left(1-g_{1}^{2}\right) \\
-f_{1}\left(1+g_{1}^{2}\right)
\end{array}\right) d u+\int\left(\begin{array}{c}
2 \omega_{2} g_{2} \\
f_{2}\left(1-g_{2}^{2}\right) \\
f_{2}\left(1+g_{2}^{2}\right)
\end{array}\right) d v
$$

is a timelike surface with mean curvature identically 0 (i.e. timelike minimal surface). Conversely, any timelike minimal surface can be described in this manner. 
The timelike minimal surfaces given by $\left(f_{1}(u), g_{1}(u)\right)=\left(1-u^{-4}, u\right),\left(f_{2}(v)\right.$, $\left.g_{2}(v)\right)=\left(1-v^{-4}, v\right)$ are called Henneberg's timelike minimal surface $\mathcal{H}(u, v)$ (timelike $\mathcal{H}$, for short), where $u, v \neq 0$, in $\mathbb{E}^{2,1}$. The parametrization of timelike $\mathcal{H}$ is

$$
\left(\begin{array}{c}
u^{2}+v^{2}+\frac{1}{u^{2}}+\frac{1}{v^{2}} \\
\frac{1}{3}\left(-u^{3}-v^{3}+\frac{1}{u^{3}}+\frac{1}{v^{3}}\right)+u+v-\frac{1}{u}-\frac{1}{v} \\
\frac{1}{3}\left(-u^{3}+v^{3}-\frac{1}{u^{3}}+\frac{1}{v^{3}}\right)-u+v-\frac{1}{u}+\frac{1}{v}
\end{array}\right)=\left(\begin{array}{l}
x(u, v) \\
y(u, v) \\
z(u, v)
\end{array}\right)
$$

where $u, v \neq 0$, with Gauss map

$$
e=\left(\frac{u v-1}{1+u v}, \frac{u+v}{1+u v}, \frac{-u+v}{1+u v}\right) .
$$

Here, $u v \neq-1$.

\section{Degrees and Classes of Henneberg Surfaces in $\mathbb{E}^{2,1}$}

For $\mathbb{E}^{2,1}$, the tangent plane at a point $(u, v)$ on a surface $f(u, v)=(x(u, v)$, $y(u, v), z(u, v))$ is given by

$$
X x+Y y-Z z+P=0,
$$

where the Gauss map is $e=(X(u, v), Y(u, v), Z(u, v))$ and $P=P(u, v)$.

In this section, $Q(x, y, z)=0$ denotes the irreducible implicit equation that spacelike or timelike Henneberg surface $\mathcal{H}$ in terms of running coordinates $x, y, z$ will satisfy. And also $\hat{Q}(a, b, c)=0$ denotes the irreducible implicit equation for spacelike or timelike $\mathcal{H}$ in terms of tangential coordinates $a, b, c$. Next, we calculate the implicit equations, degrees and classes of spacelike and timelike $\mathcal{H}$.

6.1. Degree and class of spacelike maximal Henneberg in $\mathbb{E}^{2,1}$. Taking (13), with its Gauss map, and using (18), we have

$Q(x, y, z)=64 x^{6} z^{9}+192 x^{4} y^{2} z^{9}+192 x^{2} y^{4} z^{9}+64 y^{6} z^{9}-432 x^{8} z^{6}-864 x^{6} y^{2} z^{6}-$ $768 x^{4} z^{10}+864 x^{2} y^{6} z^{6}+432 y^{8} z^{6}+768 y^{4} z^{10}-1215 x^{10} z^{3}-9963 x^{8} y^{2} z^{3}+3888 x^{8} z^{5}-$ $23814 x^{6} y^{4} z^{3}+15552 x^{6} y^{2} z^{5}+4320 x^{6} z^{7}-23814 x^{4} y^{6} z^{3}+23328 x^{4} y^{4} z^{5}-7776 x^{4} y^{2} z^{7}+$ $768 x^{4} z^{9}-9963 x^{2} y^{8} z^{3}+15552 x^{2} y^{6} z^{5}-7776 x^{2} y^{4} z^{7}-4608 x^{2} y^{2} z^{9}+2304 x^{2} z^{11}-$ $1215 y^{10} z^{3}+3888 y^{8} z^{5}+4320 y^{6} z^{7}+768 y^{4} z^{9}+2304 y^{2} z^{11}-729 x^{12}+4374 x^{10} z^{2}+$ $2187 x^{8} y^{4}+13122 x^{8} y^{2} z^{2}+7776 x^{8} z^{4}+8748 x^{6} y^{4} z^{2}+62208 x^{6} y^{2} z^{4}-52032 x^{6} z^{6}-$ $2187 x^{4} y^{8}-8748 x^{4} y^{6} z^{2}-10560 x^{4} y^{2} z^{6}-5376 x^{4} z^{8}-13122 x^{2} y^{8} z^{2}-62208 x^{2} y^{6} z^{4}+$ $10560 x^{2} y^{4} z^{6}-4608 x^{2} z^{10}+729 y^{12}-4374 y^{10} z^{2}-7776 y^{8} z^{4}+52032 y^{6} z^{6}+5376 y^{4} z^{8}+$ $4608 y^{2} z^{10}+8748 x^{10} z-26244 x^{8} y^{2} z-69120 x^{8} z^{3}+17496 x^{6} y^{4} z+89856 x^{6} z^{5}+17496 x^{4} y^{6} z+$ $511488 x^{4} y^{4} z^{3}-573696 x^{4} y^{2} z^{5}+162816 x^{4} z^{7}-26244 x^{2} y^{8} z-573696 x^{2} y^{4} z^{5}+276480 x^{2} y^{2} z^{7}-$ $27648 x^{2} z^{9}+8748 y^{10} z-69120 y^{8} z^{3}+89856 y^{6} z^{5}+162816 y^{4} z^{7}-27648 y^{2} z^{9}-9720 x^{10}+$ $79704 x^{8} y^{2}+31104 x^{8} z^{2}-190512 x^{6} y^{4}-248832 x^{6} y^{2} z^{2}+179712 x^{6} z^{4}+190512 x^{4} y^{6}+$ $1147392 x^{4} y^{2} z^{4}-595968 x^{4} z^{6}-79704 x^{2} y^{8}+248832 x^{2} y^{6} z^{2}-1147392 x^{2} y^{4} z^{4}+55296 x^{2} z^{8}+$ $9720 y^{10}-31104 y^{8} z^{2}-179712 y^{6} z^{4}+595968 y^{4} z^{6}-55296 y^{2} z^{8}+62208 x^{8} z-248832 x^{6} y^{2} z$ $-416256 x^{6} z^{3}+373248 x^{4} y^{4} z+84480 x^{4} y^{2} z^{3}+651264 x^{4} z^{5}-248832 x^{2} y^{6} z+84480 x^{2} y^{4} z^{3}-$ $1105920 x^{2} y^{2} z^{5}+62208 y^{8} z+110592 x^{2} z^{7}-416256 y^{6} z^{3}+651264 y^{4} z^{5}+110592 y^{2} z^{7}+$ 
$248832 x^{4} y^{2} z^{2}-27648 x^{8}+55296 x^{6} y^{2}+138240 x^{6} z^{2}-86016 x^{4} z^{4}-248832 x^{2} y^{4} z^{2}-$ $55296 x^{2} y^{6}-221184 x^{2} z^{6}+27648 y^{8}-138240 y^{6} z^{2}+86016 y^{4} z^{4}-32768 y^{6}+221184 y^{2} z^{6}+$ $49152 x^{4} z^{3}+294912 x^{2} y^{2} z^{3}-147456 x^{2} z^{5}+49152 y^{4} z^{3}-147456 y^{2} z^{5}+32768 x^{6}-$ $98304 x^{4} y^{2}-196608 x^{4} z^{2}+98304 x^{2} y^{4}+294912 x^{2} z^{4}+196608 y^{4} z^{2}-294912 y^{2} z$.

Its degree is $\operatorname{deg}(\mathcal{H}(u, v))=15$. Therefore, $Q(x, y, z)=0$ is an algebraic maximal surface (see Figure 1, Middle) of maximal $\mathcal{H}$. To find the class of the maximal $\mathcal{H}$, we use (13), 14) and (18). Hence, we get

$$
P(u, v)=\frac{\alpha(u, v)}{3(\lambda-1) \lambda^{2}},
$$

where $P(u, v)$ denotes the function as in equation (18) for maximal $\mathcal{H}$, and $\lambda \neq 0,1$. The inhomogeneous tangential coordinates are

$$
a=\frac{6 u \lambda^{2}}{\alpha(u, v)}, b=\frac{6 v \lambda^{2}}{\alpha(u, v)}, c=\frac{3(\lambda+1) \lambda^{2}}{\alpha(u, v)},
$$

where $\lambda=u^{2}+v^{2}, \alpha(u, v)=(\lambda+1)\left(u^{2}-v^{2}\right)\left(\lambda^{2}-4 \lambda+1\right) \neq 0$. The irreducible implicit equation $\hat{Q}(a, b, c)=0$ of spacelike $\mathcal{H}$, in the coordinates $a, b, c$ (see Figure 2 , Middle), is

$$
\hat{Q}(a, b, c)=-6 a^{4} c+4 a^{2} c^{3}+6 b^{4} c-4 b^{2} c^{3}-3 a^{4}-6 a^{2} b^{2}-3 b^{4} .
$$

Therefore, the class of spacelike maximal Henneberg is $c l(\mathcal{H}(u, v))=5$.

6.2. Degree and class of timelike minimal Henneberg in $\mathbb{E}^{2,1}$. Taking (16), with its Gauss map, and using $(18)$, we get

$Q(x, y, z)=-16 x^{9} y^{6}+48 x^{9} y^{4} z^{2}-48 x^{9} y^{2} z^{4}+16 x^{9} z^{6}+384 x^{10} y^{4}-384 x^{10} z^{4}+$ $216 x^{6} y^{8}-432 x^{6} y^{6} z^{2}+432 x^{6} y^{2} z^{6}-216 x^{6} z^{8}-2304 x^{11} y^{2}+2304 x^{11} z^{2}+768 x^{9} y^{4}+$ $4608 x^{9} y^{2} z^{2}+768 x^{9} z^{4}-4320 x^{7} y^{6}-7776 x^{7} y^{4} z^{2}+7776 x^{7} y^{2} z^{4}+4320 x^{7} z^{6}+3888 x^{5} y^{8}-$ $15552 x^{5} y^{6} z^{2}+23328 x^{5} y^{4} z^{4}-15552 x^{5} y^{2} z^{6}+3888 x^{5} z^{8}+1215 x^{3} y^{10}-9963 x^{3} y^{8} z^{2}$ $+23814 x^{3} y^{6} z^{4}-23814 x^{3} y^{4} z^{6}+9963 x^{3} y^{2} z^{8}-1215 x^{3} z^{10}-9216 x^{10} y^{2}-9216 x^{10} z^{2}+$ $10752 x^{8} y^{4}-10752 x^{8} z^{4}-104064 x^{6} y^{6}+21120 x^{6} y^{4} z^{2}+21120 x^{6} y^{2} z^{4}-104064 x^{6} z^{6}-$ $15552 x^{4} y^{8}+124416 x^{4} y^{6} z^{2}-124416 x^{4} y^{2} z^{6}+15552 x^{4} z^{8}+8748 x^{2} y^{10}-26244 x^{2} y^{8} z^{2}+$ $17496 x^{2} y^{6} z^{4}+17496 x^{2} y^{4} z^{6}-26244 x^{2} y^{2} z 8+8748 x^{2} z^{10}+1458 y^{12}-4374 y^{8} z^{4}+$ $4374 y^{4} z^{8}-1458 z^{12}+110592 x^{9} y^{2}-110592 x^{9} z^{2}+651264 x^{7} y^{4}-1105920 x^{7} y^{2} z^{2}+$ $651264 x^{7} z^{4}-359424 x^{5} y^{6}-2294784 x^{5} y^{4} z^{2}+2294784 x^{5} y^{2} z^{4}+359424 x^{5} z^{6}-276480 x^{3} y^{8}+$ $2045952 x^{3} y^{4} z^{4}-276480 x^{3} z^{8}-34992 x y^{10}-104976 x y^{8} z^{2}-69984 x y^{6} z^{4}+69984 x y^{4} z^{6}+$ $104976 x y^{2} z^{8}+34992 x z^{10}+442368 x^{8} y^{2}+442368 x^{8} z^{2}+4767744 x^{6} y^{4}-4767744 x z^{4}+$ $1437696 x^{4} y^{6}-9179136 x^{4} y^{4} z^{2}-9179136 x^{4} y^{2} z^{4}+1437696 x^{4} z^{6}-248832 x^{2} y^{8}-$ $1990656 x y^{6} z^{2}+1990656 x^{2} y^{2} z^{6}+248832 x^{2} z^{8}-77760 y^{10}-637632 y^{8} z^{2}-1524096 y^{6} z^{4}-$ $1524096 y^{4} z^{6}-637632 y^{2} z^{8}-77760 z^{10}-1769472 x^{7} y^{2}+1769472 x^{7} z^{2}+10420224 x^{5} y^{4}+$ $17694720 x^{5} y^{2} z^{2}+10420224 x^{5} z^{4}+6660096 x^{3} y^{6}+1351680 x^{3} y^{4} z^{2}-1351680 x^{3} y^{2} z^{4}-$ $6660096 x^{3} z^{6}+995328 x y^{8}+3981312 x y^{6} z^{2}+5971968 x y^{4} z^{4}+3981312 x y^{2} z^{6}+995328 x z^{8}-$ $7077888 x^{6} y^{2}-7077888 x^{6} z^{2}+2752512 x^{4} y^{4}-2752512 x^{4} z^{4}+4423680 x^{2} y^{6}-7962624 x^{2} y^{4} z^{2}-$ $7962624 x^{2} y^{2} z^{4}+4423680 x^{2} z^{6}+884736 y^{8}+1769472 y^{6} z^{2}-1769472 y^{2} z^{6}-884736 z^{8}+$ 
$9437184 x^{5} y^{2}-9437184 x^{5} z^{2}+3145728 x^{3} y^{4}-18874368 x^{3} y^{2} z^{2}+3145728 x^{3} z^{4}+$ $37748736 x^{4} y^{2}+37748736 x^{4} z^{2}+25165824 x^{2} y^{4}-25165824 x^{2} z^{4}+4194304 y^{6}+12582912 y^{4} z^{2}+$ $12582912 y^{2} z^{4}+4194304 z^{6}$.

Its degree is $\operatorname{deg}(\mathcal{H}(u, v))=15$. Hence, $Q(x, y, z)=0$ is an algebraic timelike minimal surface (see Figure 1, Right) of timelike $\mathcal{H}$. To find the class of timelike $\mathcal{H}$, we use 16, (17) and $(18)$. Therefore, we obtain

$$
P(u, v)=\frac{\hat{\alpha}(u, v)}{3(u v+1) u^{2} v^{2}},
$$

where $u v \neq-1, u, v \neq 0, P(u, v)$ denotes the function as in equation (18) for timelike $\mathcal{H}$. So, the inhomogeneous tangential coordinates are

$$
a=\frac{3 u^{2} v^{2}(u v-1)}{\hat{\alpha}(u, v)}, b=\frac{3(u+v) u^{2} v^{2}}{\hat{\alpha}(u, v)}, c=\frac{3(v-u) u^{2} v^{2}}{\hat{\alpha}(u, v)},
$$

where $\hat{\alpha}(u, v)=-(u v-1)\left(u^{2} v^{2}+4 u v+1\right)\left(u^{2}+v^{2}\right) \neq 0$. Then, the irreducible implicit equation $\hat{Q}(a, b, c)=0$ for timelike $\mathcal{H}$ in terms of inhomogeneous tangential coordinates (see Figure 2, Right) is

$$
\hat{Q}(a, b, c)=-8 a^{3} b^{2}-8 a^{3} c^{2}-12 a b^{4}+12 a c^{4}-3 b^{4}+6 b^{2} c^{2}-3 c^{4} .
$$

Finally, the class of timelike minimal Henneberg is $c l(\mathcal{H}(u, v))=5$.
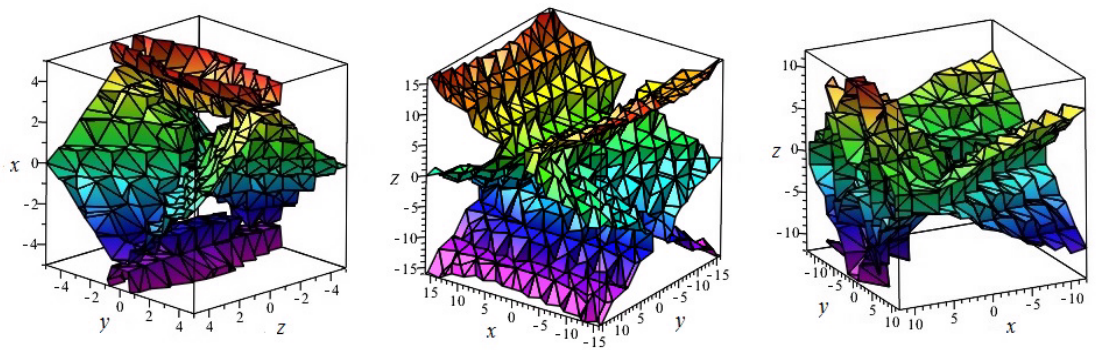

Figure 1. Henneberg algebraic surfaces $Q(x, y, z)=0$ in cartesian coordinates Left : Euclidean minimal, Middle: spacelike maximal, Right: timelike minimal

\section{Integral Free Forms in $\mathbb{E}^{2,1}$}

We reveal integral free form of the Weierstrass representation for spacelike maximal surfaces (resp. timelike minimal surfaces) in Minkowski space $\mathbb{E}^{2,1}$. 

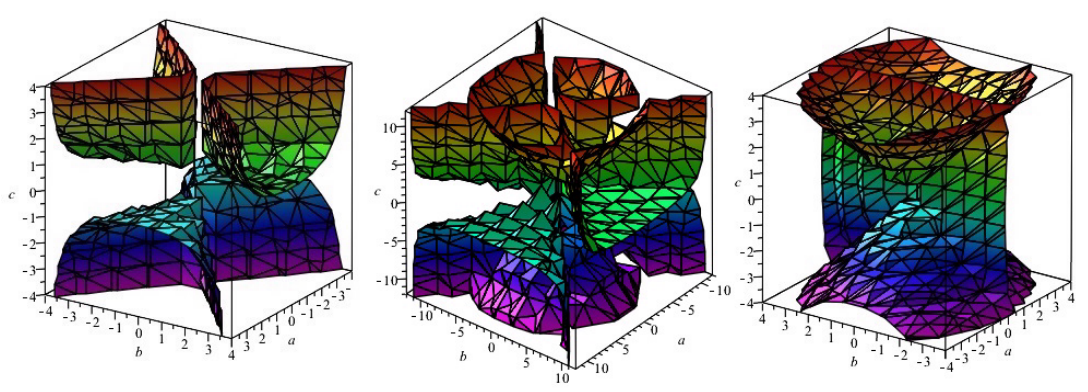

Figure 2. Henneberg algebraic surfaces $\hat{Q}(a, b, c)=0$ in inhomogeneous tangential coordinates, Left : Euclidean minimal, Middle: spacelike maximal, Right: timelike minimal

\subsection{Integral free form for spacelike maximal Henneberg.}

Proposition 11. The integral free form of the Weierstrass representation for spacelike maximal surfaces (12) in $\mathbb{E}^{2,1}$ is as follows

$$
\left(\begin{array}{l}
x \\
y \\
z
\end{array}\right)=\operatorname{Re}\left(\begin{array}{c}
\left(1+w^{2}\right) \Psi^{\prime \prime}(w)-2 w \Psi^{\prime}(w)+2 \Psi(w) \\
i\left(1-w^{2}\right) \Psi^{\prime \prime}(w)+2 i w \Psi^{\prime}(w)-2 i \Psi(w) \\
2 w \Psi^{\prime \prime}(w)-2 \Psi^{\prime}(w)
\end{array}\right) \equiv \operatorname{Re}\left(\begin{array}{c}
f_{1}(w) \\
f_{2}(w) \\
f_{3}(w)
\end{array}\right)
$$

where algebraic function $\Psi(w)$ and the functions $f_{i}(w)$ are connected by the relation

$$
\Psi(w)=\frac{1}{4}\left(w^{2}+1\right) f_{1}(w)-\frac{i}{4}\left(w^{2}-1\right) f_{2}(w)-\frac{1}{2} w f_{3}(w)
$$

for $w \in \mathbb{C}$.

Integral free form is suitable for algebraic maximal surfaces. Therefore, we obtain following:

Corollary 12. The algebraic function

$$
\Psi(w)=\frac{w^{4}+1}{6 w}
$$

leads to Henneberg spacelike maximal surface, where $w \neq 0$.

\subsection{Integral free form for timelike minimal Henneberg.}

Proposition 13. The integral free form of the Weierstrass-type representation for timelike minimal surfaces (15) in $\mathbb{E}^{2,1}$ is as follows

$$
\left(\begin{array}{l}
x \\
y \\
z
\end{array}\right)=\left(\begin{array}{c}
2 u \Omega^{\prime \prime}(u)-2 \Omega^{\prime}(u) \\
\left(1-u^{2}\right) \Omega^{\prime \prime}(u)+2 u \Omega^{\prime}(u)-2 \Omega(u) \\
-\left(1+u^{2}\right) \Omega^{\prime \prime}(u)+2 u \Omega^{\prime}(u)-2 \Omega(u)
\end{array}\right)
$$




$$
+\left(\begin{array}{c}
2 v \Theta^{\prime \prime}(v)-2 \Theta^{\prime}(v) \\
\left(1-v^{2}\right) \Theta^{\prime \prime}(v)+2 v \Theta^{\prime}(v)-2 \Theta(v) \\
\left(1+v^{2}\right) \Theta^{\prime \prime}(v)-2 v \Theta^{\prime}(v)+2 \Theta(v)
\end{array}\right) \equiv\left(\begin{array}{c}
h_{1}(u)+k_{1}(v) \\
h_{2}(u)+k_{2}(v) \\
h_{3}(u)+k_{3}(v)
\end{array}\right),
$$

where algebraic function $\Phi(u, v):=\Omega(u)+\Theta(v)$ and the functions $h_{i}(u)+k_{i}(v)$ are connected by the relation

$$
\begin{aligned}
\Phi(u, v)= & -\frac{1}{2} u h_{1}(u)+\frac{1}{4}\left(u^{2}-1\right) h_{2}(u)-\frac{1}{4}\left(u^{2}+1\right) h_{3}(u) \\
& -\frac{1}{2} v k_{1}(v)+\frac{1}{4}\left(v^{2}-1\right) k_{2}(v)+\frac{1}{4}\left(v^{2}+1\right) k_{3}(v)
\end{aligned}
$$

for $u, v \in \mathbb{R}$.

Integral free form is suitable for algebraic timelike minimal surfaces. Finally, we get the following result:

Corollary 14. The algebraic function

$$
\Phi(u, v)=\frac{v\left(u^{4}+1\right)+u\left(v^{4}+1\right)}{6 u v}
$$

leads to Henneberg timelike minimal surface, where $u, v \neq 0$.

Acknowledgement The authors would like to thank the referees for their valuable suggestions and critical remarks for improving this paper.

\section{REFERENCES}

[1] Fomenko A.T., Tuzhilin A.A., Elements of the Geometry and Topology of Minimal Surfaces in Three-Dimensional Space, Translated from the Russian by E.J.F. Primrose. Translations of Mathematical Monographs, 93. American Math. Soc., Providence, RI, 1991.

[2] Fujimori S., Saji K., Umehara M., Yamada K., Singularities of maximal surfaces, Math. Z. 259 (2008) 827-848.

[3] Gray A., Salamon S., Abbena E., Modern Differential Geometry of Curves and Surfaces with Mathematica, Third ed. Chapman \& Hall/CRC Press, Boca Raton, 2006.

[4] Henneberg L., Über salche minimalfläche, welche eine vorgeschriebene ebene curve sur geodätishen line haben. Doctoral Dissertation, Eidgenössisches Polythechikum, Zürich, 1875.

[5] Henneberg L., Über diejenige minimalfäche, welche die Neil'sche Paralee zur ebenen geodätischen line hat, Vierteljschr Natuforsch, Ges. Zürich, 21 (1876) 66-70.

[6] Henneberg L., Bestimmung der neidrigsten Classenzahl der algebraischen Minimalflächen. Annali di Matem. Pura Appl. 9 (1878) 54-57.

[7] Inoguchi J., Lee S., Null curves in Minkowski 3-space, Int. Electron. J. Geom., 1, No. 2 (2008) $40-83$.

[8] Kobayashi O., Maximal surfaces in the 3-dimensional Minkowski space $\mathbb{L}^{3}$, Tokyo J. Math. 6, No. 2 (1983) 297-309.

[9] Magid M., Timelike surfaces in Lorentz 3-space with prescribed mean curvature and Gauss map, Hokkaido Math. J. 20, No. 3 (1991) 447-464.

[10] Nitsche J.C.C., Lectures on Minimal Surfaces. Vol. 1. Introduction, Fundamentals, Geometry and Basic Boundary Value Problems, Cambridge Un. Press, Cambridge, 1989.

[11] Spivak M., A Comprehensive Introduction to Differential Geometry, Vol. IV. Third edition. Publish or Perish, Inc., Houston, Texas, 1999. 
[12] Umehara M., Yamada K., Maximal surfaces with singularities in Minkowski space, Hokkaido Math. J. 35, 1 (2006) 13-40.

[13] Weierstrass K., Untersuchungen über die Flächen, deren mittlere Krümmung überall gleich Null ist, Monatsber. d. Berliner Akad. (1866) 612-625.

[14] Weierstrass K., Über die analytische Darstellbarkeit sogenannter willkürlicher Functionen einer reellen Veränderlichen, Sitzungsberichte der Akademie zu Berlin (An expanded version of this paper with ten additional pages appeared in Weierstrass, Mathematische Werke, Mayer and Müller, Berlin, Vol. 3, (1903), 1-37), (1885), 633-639 and 789-805.

Current address: Erhan Güler (Corresponding author): Bartın University, Faculty of Science, Department of Mathematics, 74100 Bartin, Turkey.

E-mail address: eguler@bartin.edu.tr ergler@gmail.com

ORCID Address: https://orcid.org/0000-0003-3264-6239

Current address: Vahit Zambak: Bartın University, Graduate School of Natural and Applied Sciences, Mathematics Science, 74100 Bartın, Turkey.

E-mail address: vhtzmbk@gmail.com

ORCID Address: https://orcid.org/0000-0002-0492-832X 